\title{
The Dyer-Roeder distance-redshift relation in inhomogeneous universes
}

\author{
E. Mörtsell` \\ Department of Physics, Stockholm University, 10691 Stockholm, Sweden \\ Received 25 September / Accepted 16 November 2001

\begin{abstract}
Using Monte-Carlo methods, we determine the best-fit value of the homogeneity parameter $\alpha$ in the Dyer-Roeder distance-redshift relation for a variety of redshifts, inhomogeneity models and cosmological parameter values. The relation between $\alpha$ and the fraction of compact objects, $f_{\mathrm{p}}$, is found to be approximately linear. This relation can be parametrized with reasonable accuracy for all cases treated in this paper by $1-\alpha=a \cdot f_{\mathrm{p}}$, where $a \approx 0.6$
\end{abstract}

Key words. cosmology: theory - gravitational lensing

\section{Introduction}

Assuming the validity of the cosmological principle, i.e., that the Universe is isotropic and homogeneous, we can apply the Robertson-Walker (RW) metric to the field equations of general relativity to derive a relation of cosmological distance measures to redshift for various cosmological parameter values. However, we know that our Universe is very far from homogeneous on scales smaller than galaxy clusters. It is generally assumed that this does not affect the large scale expansion rate of the Universe. Still, inhomogeneities will affect measured distances through the effect of gravitational lensing. There will be different amounts of matter along different linesof-sight, causing different amounts of focusing of the lightrays. It is not possible to obtain exact solutions of the field equations for general inhomogeneous models, thus one is referred to numerical simulations to compute gravitational lensing effects on distance measurements.

Sometimes, we would like to be able to use simpler methods to compute at least approximate distances. The Dyer-Roeder (DR) distance-redshift relation (Dyer \& Roeder 1973) assumes that the expansion rate of the Universe is governed by the total matter density whereas the focusing of light is only affected by a fraction $\alpha$ of the total matter density. The DR distance thus contains an additional parameter, namely the homogeneity-parameter, $\alpha$. The approximation should be fair if a fraction $1-\alpha$ of the matter density is in very compact objects and the light-ray travels far from all matter accumulations, i.e., if one can neglect the effect of gravitational lensing.
In this paper we investigate properties of the DR distance-redshift relation by comparing with numerical simulations of the distance-redshift relation in inhomogeneous universes, including the effect from gravitational lensing. More specifically, we compute the best fit value of the homegeneity-parameter $\alpha$ for different cosmologies and inhomogeneity models. These values can be used, e.g., with some of the publicly available routines for computing cosmological distances (Kayser et al. 1995).

In an earlier study using inhomogeneity models derived from $N$-body simulations, Tomita (1998) has found the best-fit value of $\alpha$ to be close to one in most cases, with a dispersion in $\alpha$ dependent on the cosmological model, the physical radius of the inhomogenities, the redshift and separation of light-rays. This paper is complementary in the respect that we study the effect of including pointlike compact objects on the propagation of infinitesimal light-rays.

\section{Method and results}

In Fig. 1, we have plotted the DR angular-diameter distance in units of the Hubble-length for different values of the homogeneity-parameter $\alpha$. The larger the value of $\alpha$, the larger the amount of focusing of the light-rays. Thus, the angular-diameter distance decreases monotonically with $\alpha$, i.e., it increases with the "inhomogeneityparameter", $1-\alpha$. Note also that there is a simple relation between the luminosity distance, $d_{\mathrm{L}}$, and the angular-diameter distance $d_{\mathrm{A}}$,

$d_{\mathrm{L}}=(1+z)^{2} \cdot d_{\mathrm{A}}$.

\footnotetext{
* e-mail: edvard@physto.se
} 


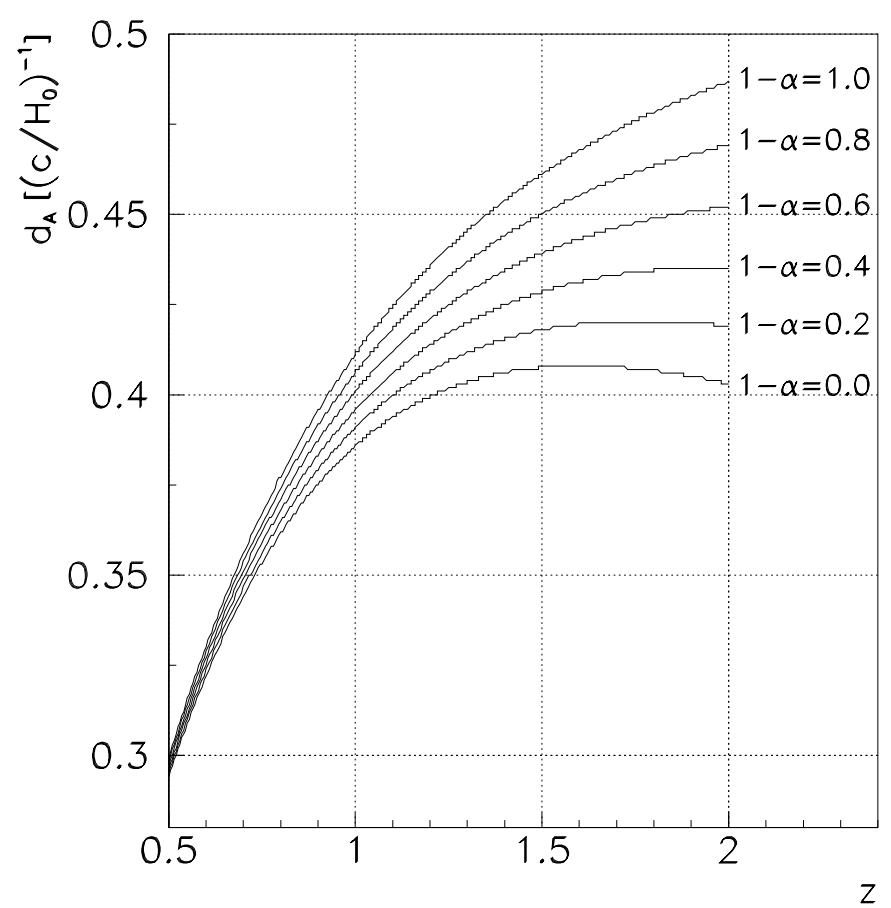

Fig. 1. The Dyer-Roeder angular-diameter distance in units of the Hubble-length for different values of the homogeneityparameter $\alpha$ and $\left(\Omega_{\mathrm{M}}, \Omega_{\Lambda}\right)=(0.3,0.7)$.

Using the simulation package SNOC (Bergström et al. 2000), we have used light-ray tracing to obtain angulardiameter distances with different inhomogeneity models and different values of the cosmological parameters. Gravitational lensing effects are calculated by integrating the geodesic deviation equation through a number of consecutive cells between the observer and the source. In each cell, we can specify the matter distribution governing the deviation. For more details of the method, see Holz \& Wald (1998) and Bergström et al. (2000). In inhomogeneous models, there will not be a one-to-one correlation between the redshift and the distance since gravitational lensing will cause a dispersion in the Hubble diagram. Figure 2 shows one simulated data set of angular-diameter distances together with the DR angular-diameter distance for three different values of $\alpha$. Using $\chi^{2}$-tests, we determine the best-fit $\alpha$-value for each of our simulated data sets.

The case closest to the premises of the derivation of the DR distance-redshift relation is the case with one component of the matter density homogeneously distributed and another in very compact objects, e.g., point-masses.

A perhaps more realistic model of our Universe has one part of the total matter density in compact objects and another part in some smooth galaxy dark matter halo model, e.g., the Navarro-Frenk-White (NFW) density profile (Navarro et al. 1997). The compact component could, e.g., be dark matter in the form of massive compact halo objects (MACHOs) where the halo fraction deduced for the Milky Way from microlensing along the line of sight to the Large Magellanic Cloud, is of the order of $20 \%$

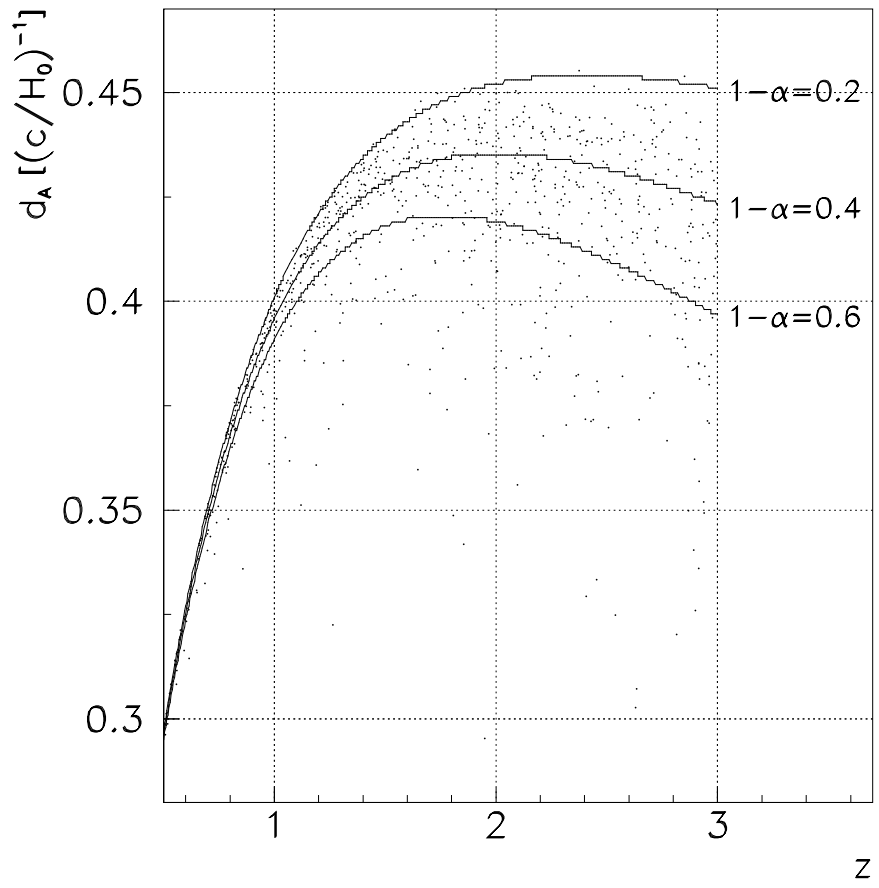

Fig. 2. The dispersion in $d_{\mathrm{A}}$ for $\left(\Omega_{\mathrm{M}}, \Omega_{\Lambda}\right)=(0.3,0.7)$ with a fraction 0.6 of the matter density in galactic dark matter halos and a fraction 0.4 in compact objects.

(Alcock et al. 2000). Note that the results are independent of the individual masses of the compact objects as well as their clustering properties on galaxy scales (Holz \& Wald 1998; Bergström et al. 2000). Note also that the exact parametrization of the galaxy density profile does not significantly affect the results, see Bergström et al. (2000).

We have used three different sets of cosmological parameter values; one open with $\left(\Omega_{\mathrm{M}}, \Omega_{\Lambda}\right)=(0.2,0)$ and two flat models with $\left(\Omega_{\mathrm{M}}, \Omega_{\Lambda}\right)=(0.3,0.7)$ and $\left(\Omega_{\mathrm{M}}, \Omega_{\Lambda}\right)=$ $(1,0)$, respectively.

In Figs. 3 to 5 we present results for $\left(\Omega_{\mathrm{M}}, \Omega_{\Lambda}\right)=$ $(0.3,0.7)$ with one homogeneous component and one component in point-masses. Distances are calculated for $z=1$, $z=2$ and a distribution of redshifts, $0.1<z<3$. For all models and redshifts, there is a linear relation between the fraction of the total matter density in point-masses, $f_{\mathrm{p}}$, and the inhomogeneity-parameter, $1-\alpha$. In the simulations using homogeneously distributed matter, we have added the condition that $1-\alpha=0$ for $f_{\mathrm{p}}=0$ when fitting the linear function.

In Figs. 6 to 8, results for the more realistic case with one part of the total matter density in point-masses and another in dark matter halos parametrized by the NFW density profile are presented. Distances are calculated in a broad redshift interval $0.1<z<3$.

\section{Discussion}

For light-rays passing far from all point-masses, we expect the best fit value of $\alpha$ to be given by $1-\alpha \approx f_{\mathrm{p}}$ where $f_{\mathrm{p}}$ is the fraction of the total matter density in 


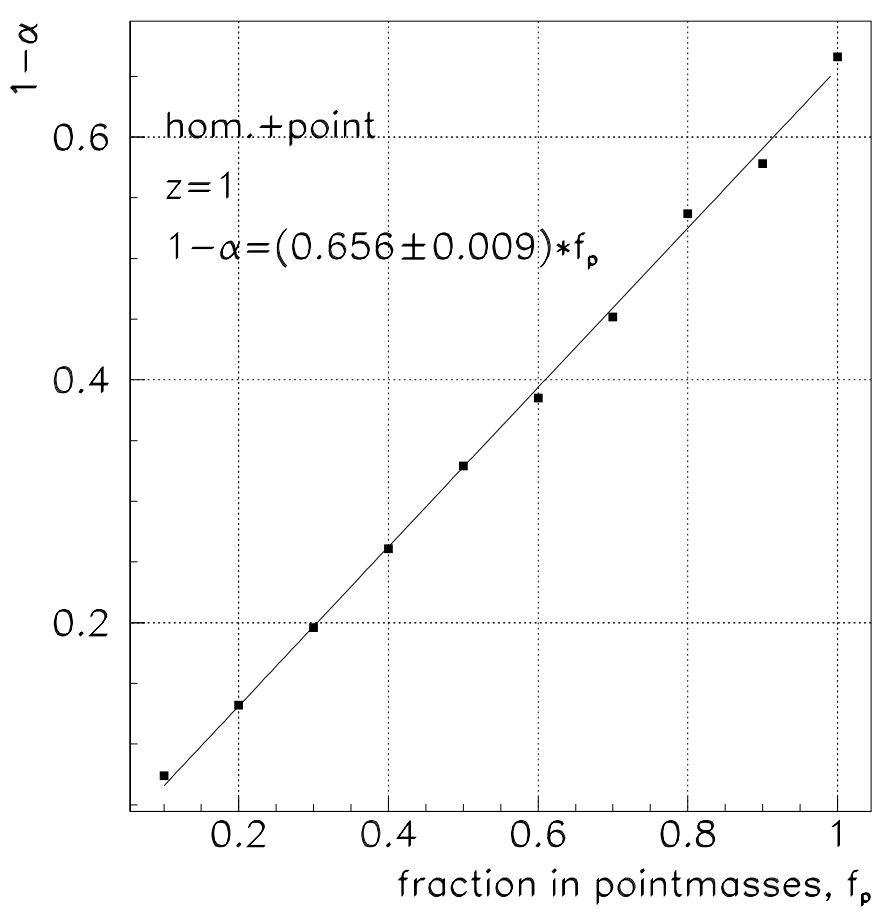

Fig. 3. Results for the homogeneity-parameter $\alpha$ for the case with one homogeneous component and one component in point-masses for $z=1$ and $\left(\Omega_{\mathrm{M}}, \Omega_{\Lambda}\right)=(0.3,0.7)$.

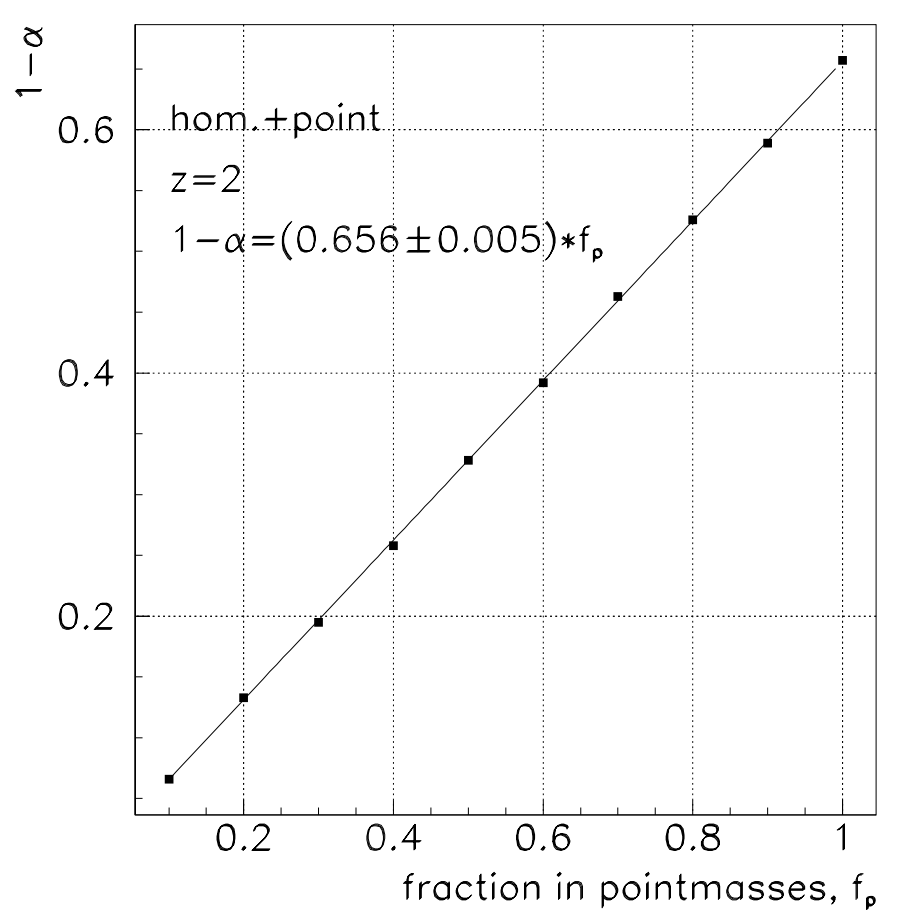

Fig. 4. Results for the homogeneity-parameter $\alpha$ for the case with one homogeneous component and one component in point-masses for $z=2$ and $\left(\Omega_{\mathrm{M}}, \Omega_{\Lambda}\right)=(0.3,0.7)$.

point-masses. Due to the magnification from lensing we expect that on average, $1-\alpha<f_{\mathrm{p}}$. It is evident from Figs. $3-5$ that the relation between $\alpha$ and $f_{\mathrm{p}}$ is independent of redshift. However, from Figs. 6-8, we can see that there is a weak cosmology dependence. We can understand

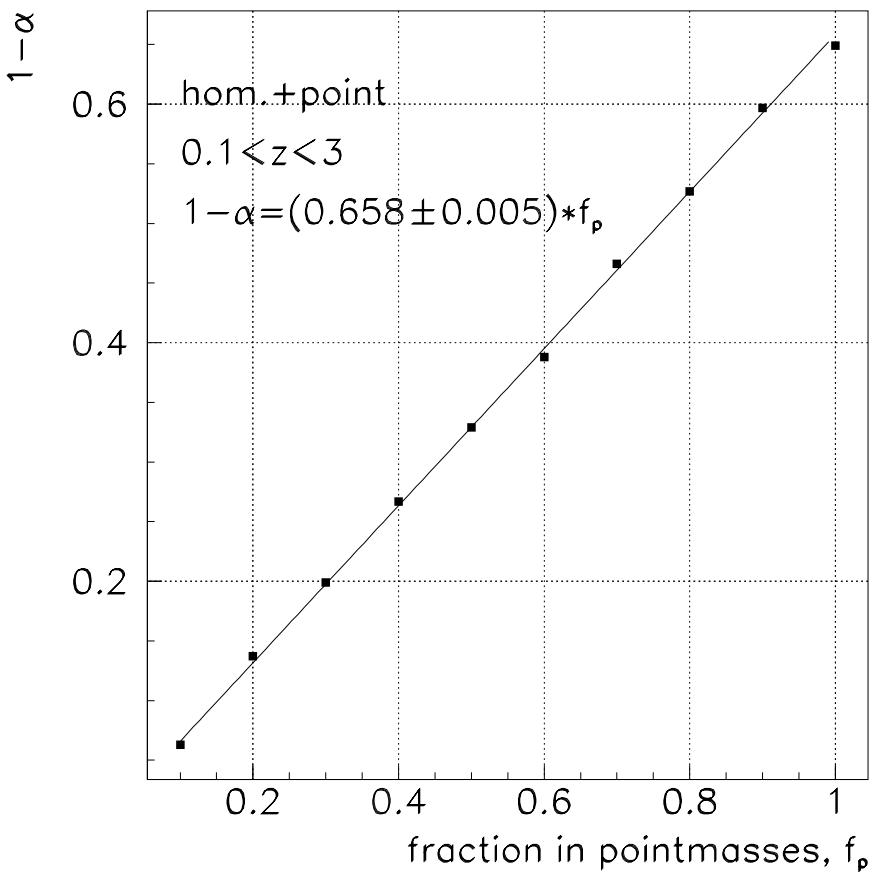

Fig. 5. Results for the homogeneity-parameter $\alpha$ for the case with one homogeneous component and one component in point-masses for $0.1<z<3$ and $\left(\Omega_{\mathrm{M}}, \Omega_{\Lambda}\right)=(0.3,0.7)$.

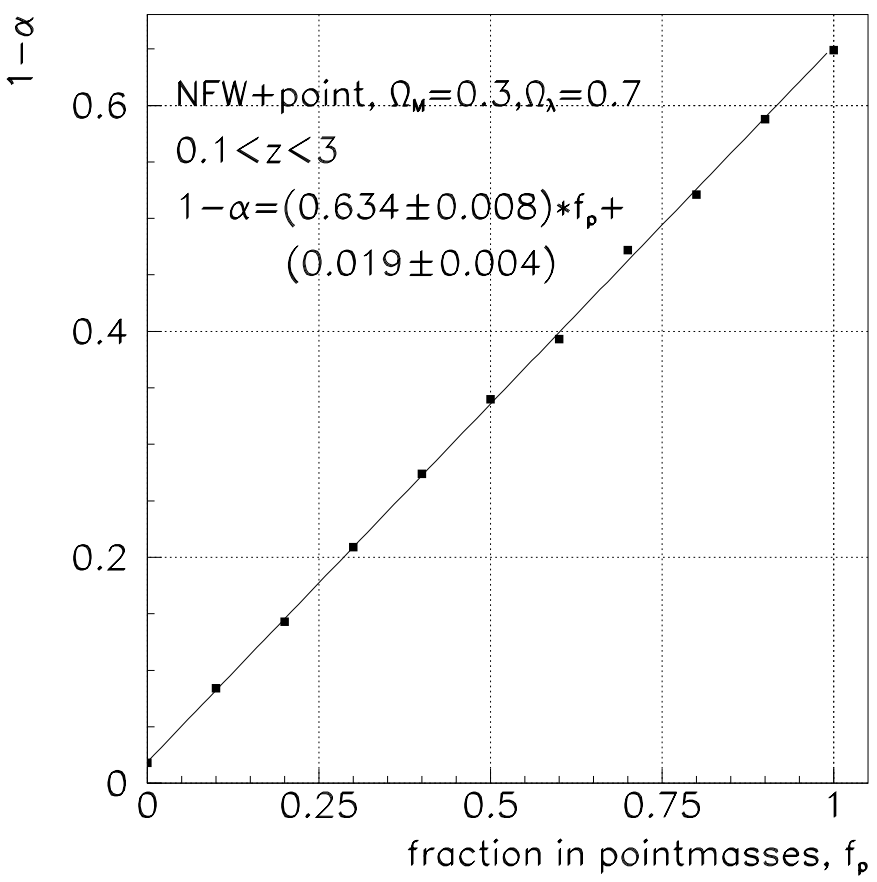

Fig. 6. Results for the homogeneity-parameter $\alpha$ for the case with one component with the NFW density profile and one in point-masses for $0.1<z<3$ and $\left(\Omega_{\mathrm{M}}, \Omega_{\Lambda}\right)=(0.3,0.7)$.

this effect from the fact that lensing effects should, for a given redshift, be roughly proportional to the distance travelled by the light-ray as well as the mass of the lenses. In Fig. 9, the number of cells traversed in the ray-tracing simulations are presented as a function of redshift for the three different cosmologies used in this paper. Since the number of cells is proportional to the distance travelled, 


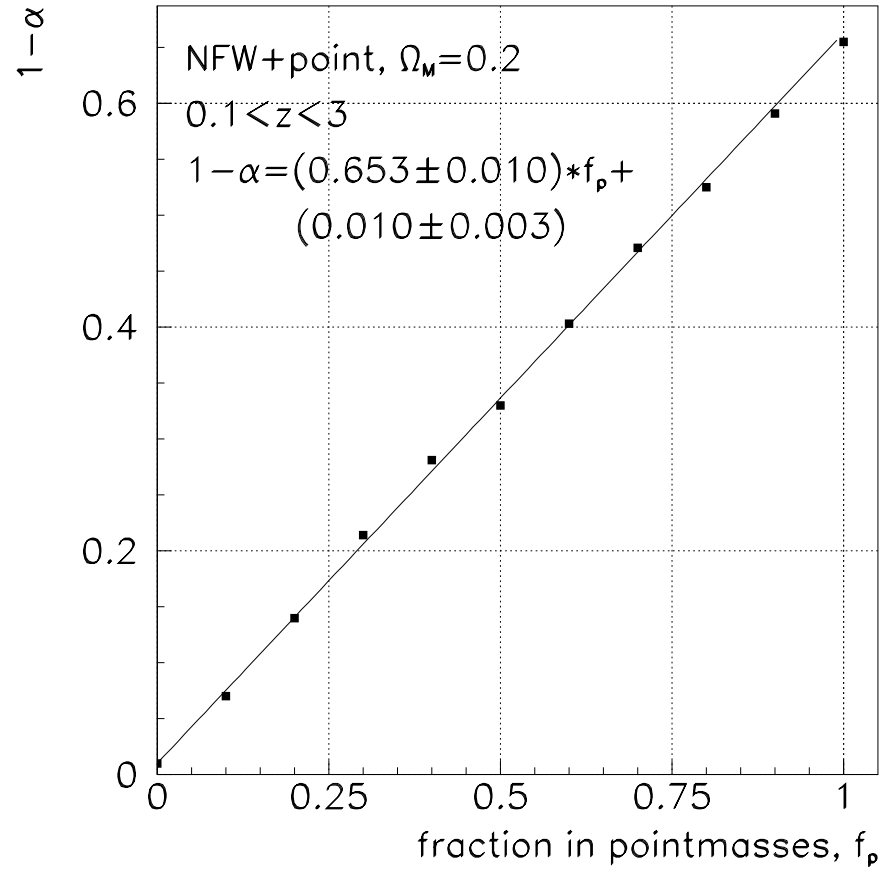

Fig. 7. Results for the homogeneity-parameter $\alpha$ for the case with one component with the NFW density profile and one in point-masses for $0.1<z<3$ and $\left(\Omega_{\mathrm{M}}, \Omega_{\Lambda}\right)=(0.2,0)$.

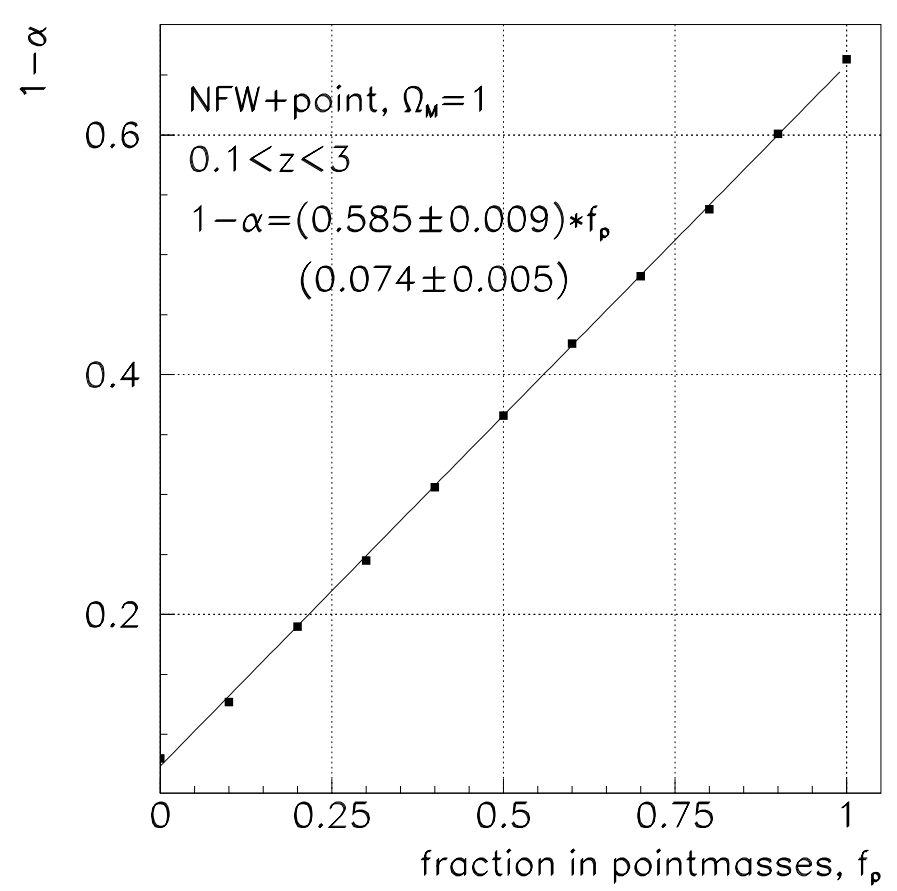

Fig. 8. Results for the homogeneity-parameter $\alpha$ for the case with one component with the NFW density profile and one in point-masses for $0.1<z<3$ and $\left(\Omega_{\mathrm{M}}, \Omega_{\Lambda}\right)=(1,0)$.

we see that the $\left(\Omega_{\mathrm{M}}, \Omega_{\Lambda}\right)=(0.3,0.7)$ cosmology yields the farthest distance, followed by the $\left(\Omega_{\mathrm{M}}, \Omega_{\Lambda}\right)=(0.2,0)$ and the $\left(\Omega_{\mathrm{M}}, \Omega_{\Lambda}\right)=(1,0)$ case. However, since the mass in lenses is proportional to $\Omega_{\mathrm{M}}$, we expect that we will have the largest lensing effects for $\left(\Omega_{\mathrm{M}}, \Omega_{\Lambda}\right)=(1,0)$ followed by $\left(\Omega_{\mathrm{M}}, \Omega_{\Lambda}\right)=(0.3,0.7)$ and $\left(\Omega_{\mathrm{M}}, \Omega_{\Lambda}\right)=(0.2,0)$, as is confirmed by Figs. $6-8$.
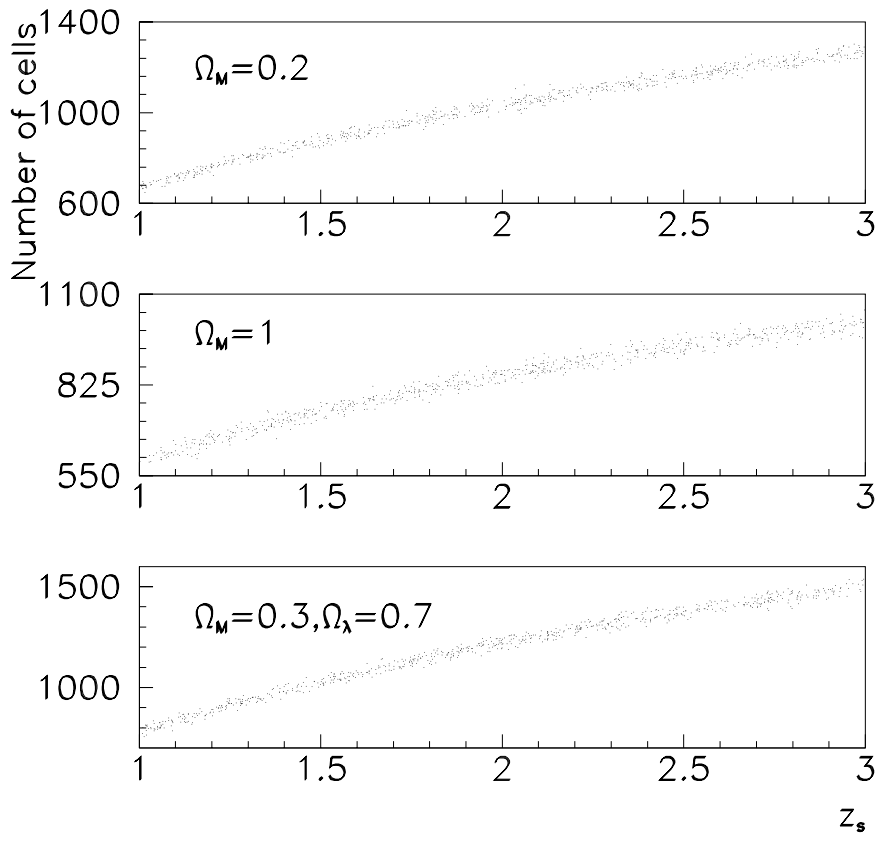

Fig. 9. Number of cells traversed in the ray-tracing simulations for $\left(\Omega_{\mathrm{M}}, \Omega_{\Lambda}\right)=(0.2,0)$ (top), $\left(\Omega_{\mathrm{M}}, \Omega_{\Lambda}\right)=(1,0)$ (middle) and $\left(\Omega_{\mathrm{M}}, \Omega_{\Lambda}\right)=(0.3,0.7)$ (bottom).
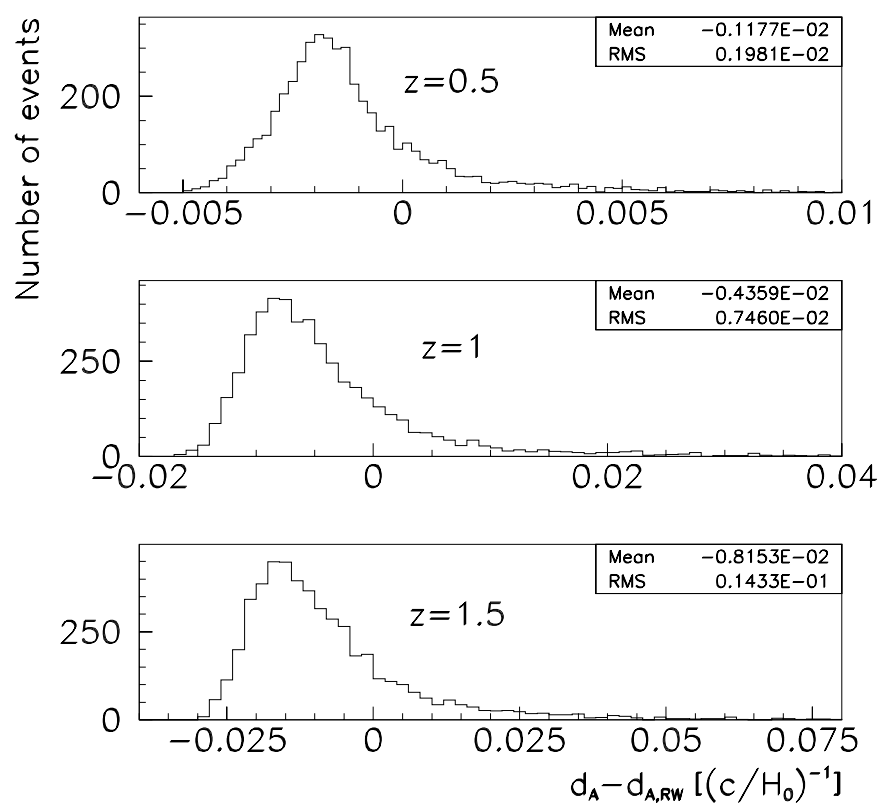

Fig. 10. The dispersion in $d_{\mathrm{A}}$ for the NFW case with $f_{\mathrm{p}}=0$ and $\left(\Omega_{\mathrm{M}}, \Omega_{\Lambda}\right)=(0.3,0.7)$ for $z=0.5, z=1$ and $z=1.5$.

We can estimate the dispersion in $\alpha$ from Fig. 2 to be $\sigma_{\alpha} \approx 0.2$. However, since we are measuring distances, the dispersion in distance at a given redshift is perhaps of greater interest. In Fig. 10, the dispersion in $d_{\mathrm{A}}$ for $f_{\mathrm{p}}=0.2$ and $\left(\Omega_{\mathrm{M}}, \Omega_{\Lambda}\right)=(0.3,0.7)$ for $z=0.5, z=1$ and $z=1.5$ is shown. The zero-value corresponds to the value one would obtain in a homogeneous universe, the so called Robertson-Walker angular-diameter distance, $d_{\mathrm{A}, \mathrm{RW}}$. The dispersion in angular-diameter distance ( $\mathrm{rms}$ ) corresponds to $5.9 / h \mathrm{Mpc}$ for $z=0.5,22 / h \mathrm{Mpc}$ for $z=1$ and 
$43 / h$ Mpc for $z=1.5$. It is also evident from Fig. 10 that the RW value is within the rms-dispersion in all three cases. However, since the mean value is displaced from zero, the use of the RW-distance will cause systematic errors of the order 3.5/h Mpc for $z=0.5,13 / h \mathrm{Mpc}$ for $z=1$ and $24 / h$ Mpc for $z=1.5$.

\section{Summary}

We have investigated the best-fit values of the homogeneity-parameter $\alpha$ in the Dyer-Roeder distanceredshift relation. For a variety of inhomogeneity models, redshifts and cosmological parameter values, the relation between $\alpha$ and the fraction of compact objects, $f_{\mathrm{p}}$, is approximately linear and we can parametrize the relation by

$1-\alpha=a \cdot f_{\mathrm{p}}$

where $a \approx 0.6$. We expect the dispersion in the angular-diameter distance to be $5.9 / h \mathrm{Mpc}$ for $z=0.5$, $22 / h \mathrm{Mpc}$ for $z=1$ and $43 / h \mathrm{Mpc}$ for $z=1.5$ in doing this approximation. In the limit of no point-like compact objects $\left(f_{\mathrm{p}}=0\right)$, our results agree with those of Tomita (1998), namely that the best-fit value of $\alpha$ is close to one.

Acknowledgements. The author would like to thank Ariel Goobar for useful comments.

\section{References}

Alcock, C., Allsnar, R. A., Alues, D. R., et al. 2000, ApJ, 542, 281

Bergström, L., Goliath, M., Goobar, A., \& Mörtsell, E. 2000, A\&A, 358, 13

Dyer, C. C., \& Roeder, R. C. 1973, ApJ, 180, 31

Holz, D. E, \& Wald, R. M. 1998, Phys. Rev. D, 58, 063501

Kayser, R., Helbig, P., \& Schramm, T. 1995, A\&A, 318, 680

Navarro, J. F., Frenk, C. S., \& White, S. D. M. 1997, ApJ, 490, 493

Tomita, K. 1998, Prog. Theor. Phys. 100, 79 [astro-ph/9806047]; See also Tomita, K., et al. 1999, Prog. Theor. Phys. Suppl., 133, 155 [astro-ph/9904351] 